JOURNAL OF INTEGRAL EQUATIONS

AND APPLICATIONS

Volume 5, Number 4, Fall 1993

\title{
REGULARIZED SOLUTIONS OF A CAUCHY PROBLEM FOR THE LAPLACE EQUATION IN AN IRREGULAR STRIP
}

\author{
D.D. ANG, D.N. THANH AND V.V. THANH
}

\begin{abstract}
The problem of finding a harmonic function in a plane strip bounded by a straight line and a curve $C: y=\phi(x)$ with Cauchy data specified on $C$ is formulated as an integral equation of first kind. The latter is converted into an equivalent convolution equation, which is regularized via the Tikhonov method, with error estimates given.
\end{abstract}

Introduction. As first pointed out by Hadamard, the Cauchy problem for the Laplace equation is an ill-posed problem. While the Dirichlet problem for the Laplace equation is a classical well-posed problem, the needs of science and technology make it necessary in many important cases to consider, instead, the Cauchy problem for that equation. For example, one may need to determine the gravity potential in a portion of the Earth bounded by an outer surface $S_{1}$, which lies on the surface of the Earth, and an inner surface $S_{2}$ lying inside the Earth. Clearly, it is impractical, if not impossible, to measure the potential on $S_{2}$. Therefore, one should be content with measurements performed on $S_{1}$ (of the potential and of the flux). It is the case that these measurements are sufficient to determine the potential inside; this follows from uniqueness theorems for the Cauchy problem for the Laplace equation (see, e.g., [5]). However, as pointed out above, the problem is ill-posed, i.e., solutions do not always exist and, whenever they do exist, there is no continuous dependence on the given data. The reader is referred to $[\mathbf{1}, \mathbf{2}, \mathbf{3}, \mathbf{5}, \mathbf{6}, \mathbf{8}, \mathbf{9}]$ for the earlier literature on the Cauchy problem for the Laplace equation.

For numerical computations, ill-posed problems need to be regularized. A regularized solution is a stable approximate solution. An important question arises as to how close a regularized solution is to

\footnotetext{
Received by the editors on June 3, 1992 and in revised form on May 24, 1993.

AMS Subject Classification (1991). 35J05, 35R25.

Key words and phrases. Cauchy problem, Laplace equation, regularized solutions.

The work of the first author was completed with financial support from the National Basic Research Program in Natural Sciences.

Copyright (C)1993 Rocky Mountain Mathematics Consortium
} 
an exact solution, especially when the measured data is affected with noise. The question of error estimates is of importance, and in order to have specific estimates, one usually has to consider specific cases. In this paper, we are concerned with the problem of finding a function $u$, harmonic in the plane domain $D$ defined by

$$
D=\{(x, y):-\infty<x<\infty, 0<y<\phi(x)\}
$$

and continuous on $\bar{D}$, given $u, u_{x}$ and $u_{y}$ on the portion of the boundary represented by the curve $y=\phi(x)$. Here $\phi$ is of class $C^{1}$. The problem of regularizing the Cauchy problem for the Laplace equation in a rather general context was considered, e.g., in [4]; using the method of quasireversibility, the authors (loc. cit.) stabilized the problem, but no error estimates are given. In the present paper we shall use the Tikhonov method of regularization. Taking the boundary value $v(x)=u(x, 0)$ as our unknown, we shall show that if the discrepancy between the given values of $u, u_{x}, u_{y}$ on the curve $y=\phi(x)$ and their exact values is of the order $\varepsilon$ (with respect to some appropriate metric), then, assuming the exact solution $v_{0}(x)$ to be smooth (in $H^{1}(\mathbf{R})$ ), the discrepancy between the regularized solution and the exact solution $v_{0}(x)$ is of the $\operatorname{order}(\ln 1 / \varepsilon)^{-1}$ as $\varepsilon \rightarrow 0$.

Integral equation formulation and regularization. We first set some notations:

$$
\begin{aligned}
u_{x}(x, \phi(x)) & =f(x), \\
u_{y}(x, \phi(x)) & =g(x), \\
u(x, \phi(x)) & =u_{1}(x) .
\end{aligned}
$$

These functions, we recall, are given. Let us put

$$
\begin{aligned}
\Gamma(x, y ; \xi, \eta) & =-\frac{1}{2 \pi} \ln \left((x-\xi)^{2}+(y-\eta)^{2}\right)^{1 / 2} \\
G(x, y ; \xi, \eta) & =\Gamma(x, y ; \xi, \eta)-\Gamma(x, y ; \xi,-\eta)
\end{aligned}
$$

where $\Gamma$ is a fundamental solution of the Laplace equation and $G$ is the Green's function for the Laplacian corresponding to a Dirichlet condition at the boundary $y=0$.

It is sufficient to determine $u(x, 0)=v(x)$. Once this is done, $u(x, y)$ is known. We shall derive an integral equation in $v$. In order to do 


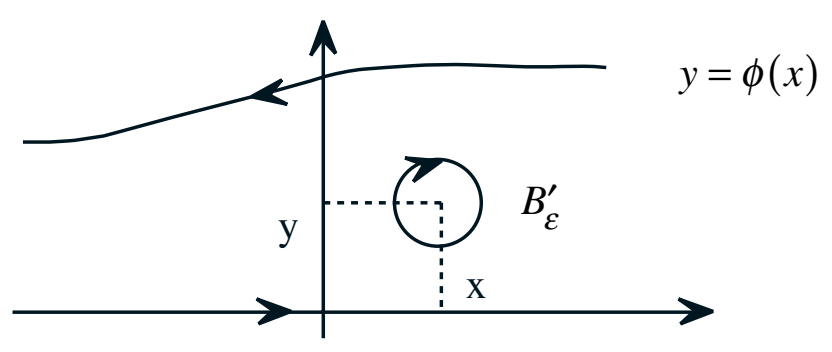

FIGURE 1.

this, we integrate Green's identity on $D_{\varepsilon}, \varepsilon>0$, where $D_{\varepsilon}=D \backslash B_{\varepsilon}^{\prime}$ and $B_{\varepsilon}^{\prime}$ : closed ball in $D$ of radius $\varepsilon$ centered at $(x, y)$, and let $\varepsilon \rightarrow 0$ (see Figure 1).

We then have, after some rearrangements

$$
\begin{aligned}
(1 / \pi) \int_{-\infty}^{\infty} \frac{y v(\xi)}{(x-\xi)^{2}+y^{2}} d \xi= & -u(x, y)-\int_{-\infty}^{\infty} G(x, y ; \xi, \phi(\xi)) f_{1}(\xi) d \xi \\
& +\int_{-\infty}^{\infty} G_{1}(x, y ; \xi, \phi(\xi)) u_{1}(\xi) d \xi, \\
& -\infty<x<\infty, 0<y<\phi(x),
\end{aligned}
$$

where $f_{1}(\xi)=g(\xi)-f(\xi) \phi^{\prime}(\xi)$ and

$$
\begin{aligned}
G_{1}(x, y ; \xi, \phi(\xi))= & G_{\eta}(x, y ; \xi, \phi(\xi))-G_{\xi}(x, y ; \xi, \phi(\xi)) \phi^{\prime}(\xi) \\
= & (1 / 2 \pi) \frac{y-\phi(\xi)-(x-\xi) \phi^{\prime}(\xi)}{(x-\xi)^{2}+(y-\phi(\xi))^{2}} \\
& +(1 / 2 \pi) \frac{y+\phi(\xi)+(x-\xi) \phi^{\prime}(\xi)}{(x-\xi)^{2}+(y+\phi(\xi))^{2}} .
\end{aligned}
$$

The foregoing calculations are purely formal. In order for them to be valid, we have to make some assumptions on the given data and on the properties of the solution to be sought. We shall, accordingly, make the following standing 
Assumptions. (i) The curve $y=\phi(x)$ is eventually straight, which means that $\phi^{\prime}(x)=0$ for large $|x|$.

(ii) $f(x), g(x)$ and $u_{1}(x)$ tend to 0 sufficiently fast, say as $1 /|x|$ as $|x| \rightarrow \infty$.

(iii) $\left(1+x^{2}\right)^{1 / 2} v(x)$ is in $L^{2}(\mathbf{R})$.

Letting $y \rightarrow \phi(x)$ in (3), we have (see [7, pp. 144-145])

(5) $\frac{1}{\pi} \int_{-\infty}^{\infty} \frac{\phi(x) v(\xi) d \xi}{(x-\xi)^{2}+\phi^{2}(x)}=-(3 / 2) u_{1}(x)$

$-\int_{-\infty}^{\infty} G(x, \phi(x) ; \xi, \phi(\xi)) \cdot f_{1}(\xi) d \xi+\int_{-\infty}^{\infty} G_{1}(x, \phi(x): \xi, \phi(\xi)) u_{1}(\xi) d \xi$,

which is an integral equation in $v(x)$. We shall convert (5) into a convolution equation.

We note that the function

$$
H(x, y)=\frac{1}{\pi} \int_{-\infty}^{\infty} \frac{y v(\xi)}{(x-\xi)^{2}+y^{2}} d \xi
$$

is harmonic in the upper right half plane $y>0$. The value $H(x, \phi(x))$ is then the right hand side of (5). As shown in the Appendix, we can calculate $(\partial H / \partial n)(x, \phi(x))$ as the limit from below of the directional derivative of the right hand side of (3) when $(x, y) \rightarrow(x, \phi(x)), \vec{n}$ being the inner unit normal to the curve $y=\phi(x)$ (Figure 2). Let

$$
\lambda(x)=H(x, \phi(x)), \quad \mu(x)=\frac{\partial H}{\partial n}-(x, \phi(x)) .
$$

Then $H(x, y)$ can be represented as a potential with densities $\lambda, \mu$ on the domain $y>\phi(x)$. 


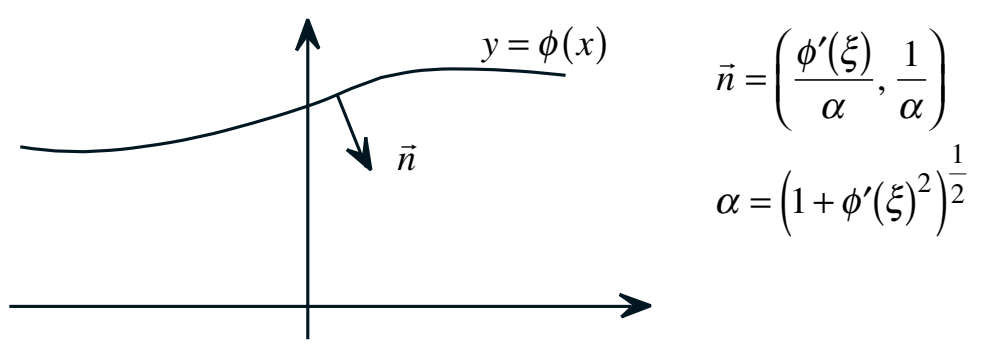

FIGURE 2.

We now write explicit expressions for $\lambda(x)$ and $\mu(x)$. We have

$$
\begin{aligned}
\lambda(x)= & -(3 / 2) u_{1}(x)-\int_{-\infty}^{\infty} G(x, \phi(x) ; \xi, \phi(\xi)) f_{1}(\xi) d \xi \\
& +\int_{-\infty}^{\infty} G_{1}(x, \phi(x) ; \xi, \phi(\xi)) u_{1}(\xi) d \xi,
\end{aligned}
$$

(6)

$$
\begin{aligned}
\mu(x)= & (3 / 2) f_{1}(x)-\alpha(x)^{-1} \int_{-\infty}^{\infty} G_{2}(x, \phi(x) ; \xi, \phi(\xi)), f_{1}(\xi) d \xi \\
& +\alpha(x)^{-1}(2 \pi)^{-1} \int_{-\infty}^{\infty} \frac{(\phi(x)+\phi(\xi)) \phi^{\prime}(x)+x-\xi}{(x-\xi)^{2}+(\phi(x)+\phi(\xi))^{2}} u_{1}^{\prime}(\xi) d \xi \\
& +(\alpha(x) 2 \pi)^{-1} \int_{-\infty}^{\infty} \frac{(\phi(x)-\phi(\xi)) \phi^{\prime}(x)+x-\xi}{(x-\xi)^{2}+(\phi(x)-\phi(\xi))^{2}} u_{1}^{\prime}(\xi) d \xi,
\end{aligned}
$$

in which

$$
\begin{aligned}
G_{2}(x, \phi(x) ; \xi, \phi(\xi))= & G_{x}(x, \phi(x) ; \xi, \phi(\xi)) \cdot \phi^{\prime}(x) \\
& -G_{y}(x, \phi(x) ; \xi, \phi(\xi)), \\
\alpha(x)= & \left(1+\phi^{\prime}(x)^{2}\right)^{1 / 2} .
\end{aligned}
$$

By (6), the functions $\lambda(x)$ and $\mu(x)$ are defined on $\mathbf{R}$ and depend continuously on $\phi(x), \phi^{\prime}(x), u_{1}(x), u_{1}^{\prime}(x), f(x)$ and $g(x)$ in the $L^{2}(\mathbf{R})$ sense.

Now, integrating Green's identity in the domain

$$
D_{R}=\{(x, y):|x|<R, \phi(x)<y<R\}
$$


and letting $R \rightarrow \infty$, we get

$$
\begin{aligned}
H(x, y)= & \int_{-\infty}^{\infty} \Gamma(x, y ; \xi, \phi(\xi)) \mu(\xi) d \xi \\
& -\int_{-\infty}^{\infty} \Gamma_{1}(x, y ; \xi, \phi(\xi)) \lambda(\xi) d \xi
\end{aligned}
$$

for $-\infty<x<\infty, y>\phi(x)$, where

$$
\begin{aligned}
G_{1}(x, y ; \xi, \phi(\xi)) & =\Gamma_{\xi}(x, y ; \xi, \phi(\xi)) \phi^{\prime}(\xi)-\Gamma_{\eta}(x, y ; \xi, \phi(\xi)) \\
& =(2 \pi)^{-1} \frac{(x-\xi) \phi^{\prime}(\xi)-(y-\phi(\xi))}{(x-\xi)^{2}+(y-\phi(\xi))^{2}} .
\end{aligned}
$$

Note that as $R \rightarrow \infty$, the integral on

$$
\begin{aligned}
C_{R}= & \{(R, y): \phi(R)<y<R\} \cup\{(-R, y): \phi(-R)<y<R\} \\
& \cup\{(x, R):-R<x<R\}
\end{aligned}
$$

tends to 0 as a consequence of our assumption on $v$ (i.e., $\left(1+x^{2}\right)^{1 / 2} v(x)$ in $L^{2}(\mathbf{R})$ ).

Evaluating $H(x, y)$ at $(x, k)$ where $k$ is a fixed number greater than $\phi(x)$ for all $x$ in $\mathbf{R}$, we have by (7)

$$
\begin{aligned}
\frac{1}{\pi} \int_{-\infty}^{\infty} \frac{k v(\xi)}{(x-\xi)^{2}+k^{2}} d \xi= & \int_{-\infty}^{\infty} \Gamma(x, k ; \xi, \phi(\xi)) \mu(\xi) d \xi \\
& -\int_{-\infty}^{\infty} \Gamma_{1}(x, k ; \xi, \phi(\xi)) \lambda(\xi) d \xi
\end{aligned}
$$

Let

$$
\begin{aligned}
F(x)= & \pi \int_{-\infty}^{\infty} \Gamma(x, k ; \xi, \phi(\xi)) \mu(\xi) d \xi \\
& -\pi \int_{-\infty}^{\infty} \Gamma_{1}(x, k ; \xi, \phi(\xi)) \lambda(\xi) d \xi
\end{aligned}
$$

Then we have a convolution integral equation in $v(\xi)$

$$
\int_{-\infty}^{\infty} k v(\xi)\left((x-\xi)^{2}+k^{2}\right)^{-1} d \xi=F(x), \quad \forall x \in \mathbf{R}
$$


which is an integral equation of first kind, and we know that this problem is ill-posed. We shall construct a family $\left(v_{\beta}\right), \beta>0$, of regularized solutions (see $[\mathbf{1 0}]$ ), and we pick a regularized solution that is "close" to the exact solution. We recall that, by regularized solution, we mean a function that is stable with respect to variations in the right hand side of (8).

We now state and prove our main result.

Theorem. Suppose the exact solution $v_{0}$ of (8) corresponding to $F_{0}$ in the right hand side is in $H^{1}(\mathbf{R})$ and let

$$
\left|F_{0}-F\right|_{2}<\varepsilon, \quad|\cdot|_{2}=L^{2}(\mathbf{R})-\text { norm } .
$$

Then there exists a regularized solution $v_{\varepsilon}$ of (8) such that

$$
\left|v_{\varepsilon}-v_{0}\right| 2 \leq K(\ln (1 / \varepsilon))^{-1} \quad \text { for } \varepsilon \rightarrow 0
$$

where $K$ is a constant depending only on the $H^{1}$-norm of $v_{0}$.

Proof. Letting $G(x)=k /\left(x^{2}+k^{2}\right)$, we have

$$
\hat{G}(t)=(\pi / 2)^{1 / 2} e^{-k|t|}
$$

where

$$
\hat{G}(t)=(2 \pi)^{-1 / 2} \int_{-\infty}^{\infty} G(x) e^{-i x t} d x .
$$

For $v$ in $L^{2}(\mathbf{R})$, we then have from (8)

$$
\hat{G}(t) \hat{v}(t)=\hat{F}(t) .
$$

Now let $v_{0} \in H^{1}(\mathbf{R})$ be the exact solution of the equation

$$
\hat{G}(t) \hat{v}_{0}(t)=\hat{F}_{0}(t), \quad \forall t \in \mathbf{R}
$$

with $F$ and $F_{0}$ in $L^{2}(\mathbf{R})$ such that

$$
\left|F-F_{0}\right|_{2}<\varepsilon
$$


For every $\beta>0$, the function

$$
\psi(t)=\hat{G}(t)\left(\beta+\hat{G}^{2}(t)\right)^{-1} \hat{F}(t)
$$

is in $L^{2}(\mathbf{R})$. Let

$$
v_{\beta}(x)=(2 \pi)^{-1 / 2} \int_{-\infty}^{\infty} \psi(t) e^{i x t} d t
$$

Then $v_{\beta} \in L^{2}(\mathbf{R})$ and, by (11), $v_{\beta}$ satisfies the equation

$$
\beta \hat{v}_{\beta}(t)+\hat{G}^{2}(t) \hat{v}_{\beta}(t)=\hat{G}(t) \hat{F}(t) \quad \forall t \in \mathbf{R}
$$

and depends continuously on $F(t)$.

We now derive error estimates. From (9) and (12), we have

$$
\begin{aligned}
\beta\left(\hat{v}_{\beta}(t)-\hat{v}_{0}(t)\right)+\hat{G}^{2}(t)\left(\hat{v}_{\beta}(t)-\hat{v}_{0}(t)\right) \\
\quad=-\beta \hat{v}_{0}(t)+\hat{G}(t)\left(\hat{F}(t)-\hat{F}_{0}(t)\right) \quad \forall t \in \mathbf{R} .
\end{aligned}
$$

We multiply both sides of $(13)$ by $\left(\overline{\hat{v}}_{\beta}(t)-\overline{\hat{v}}(t)\right)$ and then integrate on R. We have

$$
\begin{aligned}
\beta\left|\hat{v}_{\beta}-\hat{v}_{0}\right|_{2}^{2}+\left|\hat{G}\left(\hat{v}_{\beta}-\hat{v}_{0}\right)\right|_{2}^{2}= & -\int_{-\infty}^{\infty} \beta \hat{v}_{0}(t)\left(\overline{\hat{v}}(t)-\overline{\hat{v}}_{0}(t)\right) d t \\
& +\int_{-\infty}^{\infty} \hat{G}(t)\left(\hat{F}(t)-\hat{F}_{0}(t)\right)\left(\overline{\hat{v}}_{\beta}-\overline{\hat{v}}_{0}(t)\right) d t \\
\leq & \beta\left|\hat{v}_{0}\right|_{2}\left|\hat{v}_{\beta}-\hat{v}_{0}\right|_{2} \\
& +(\pi / 2)^{1 / 2} \cdot\left|\hat{F}-\hat{F}_{0}\right|_{2}\left|\hat{v}_{\beta}-\hat{v}_{0}\right|_{2} .
\end{aligned}
$$

Let $\beta=\varepsilon$ and note that $\left|\hat{F}-\hat{F}_{0}\right|_{2}=\left|F-F_{0}\right|_{2}<\varepsilon$, we have

$$
\varepsilon\left|\hat{v}_{\varepsilon}-\hat{v}_{0}\right|_{2}^{2}+\left|\hat{G}\left(\hat{v}_{\varepsilon}-\hat{v}_{0}\right)\right|_{2}^{2} \leq \varepsilon\left(\left|\hat{v}_{0}\right|_{2}+(\pi / 2)^{1 / 2}\right)\left|\hat{v}_{\varepsilon}-\hat{v}_{0}\right|_{2} .
$$

In particular

$$
\left|\hat{v}_{\varepsilon}-\hat{v}_{0}\right|_{2} \leq\left|\hat{v}_{0}\right|_{2}+(\pi / 2)^{1 / 2} .
$$


Similarly, we multiply both sides of (13) by $t^{2}\left(\overline{\hat{v}}(t)-\overline{\hat{v}}_{0}(t)\right)$ and integrate over $\mathbf{R}$. We have

$$
\begin{aligned}
\varepsilon\left|t\left(\hat{v}_{\varepsilon}-\hat{v}_{0}\right)\right|_{2}^{2}+ & \left|\hat{G} t\left(\hat{v}_{\varepsilon}-\hat{v}_{0}\right)\right|_{2}^{2} \\
= & \int_{-\infty}^{\infty}-\varepsilon \hat{v}_{0}(t) t^{2}\left(\overline{\hat{v}}_{\varepsilon}(t)-\overline{\hat{v}}_{0}(t)\right) d t \\
& +\int_{-\infty}^{\infty} t \hat{G}(t)\left(\hat{F}(t)-\hat{F}_{0}(t)\right) t\left(\overline{\hat{v}}_{\varepsilon}(t)-\overline{\hat{v}}_{0}(t)\right) d t \\
\leq & \varepsilon\left|\hat{v}_{0}^{\prime}\right|_{2}\left|t\left(\hat{v}_{\varepsilon}-\hat{v}_{0}\right)\right|_{2} \\
& +e^{-1}(\pi / 2)^{1 / 2}\left|\hat{F}-\hat{F}_{0}\right|_{2} \mid t\left(\hat{v}_{\varepsilon}-\left.\hat{v}_{0}\right|_{2}\right. \\
\leq & \varepsilon\left(\left|\hat{v}_{0}^{\prime}\right|_{2}+e^{-1}(\pi / 2)^{1 / 2} \mid t\left(\hat{v}_{\varepsilon}-\left.\hat{v}_{0}\right|_{2} .\right.\right.
\end{aligned}
$$

In particular,

$$
\left|t\left(\hat{v}_{\varepsilon}-\hat{v}_{0}\right)\right|_{2} \leq\left|\hat{v}_{0}^{\prime}\right|_{2}+e^{-1}(\pi / 2)^{1 / 2} .
$$

Since

$$
\left|v_{\varepsilon}-v_{0}\right|_{2}=\left|\hat{v}_{\varepsilon}-\hat{v}_{0}\right|_{2},
$$

and

$$
\left|v_{\varepsilon}^{\prime}-v_{0}^{\prime}\right|_{2}=\left|t\left(\hat{v}_{\varepsilon}-\hat{v}_{0}\right)\right|_{2},
$$

from (16) and (18) we have

$$
\| v_{\varepsilon}-\left.v_{0}\right|_{H^{1}}=\left|v_{\varepsilon}-v_{0}\right|_{2}+\left|v_{\varepsilon}^{\prime}-v_{0}^{\prime}\right|_{2} \leq K_{1}
$$

where $K_{1}=\max \left(\left|\hat{v}_{0}\right|_{2}+(\pi / 2)^{1 / 2},\left|\hat{v}_{0}^{\prime}\right|_{2}+e^{-1}(\pi / 2)^{1 / 2}\right)$.

We have, for any $t_{\varepsilon}>0$,

$$
\begin{aligned}
\int_{|t| \leq t_{\varepsilon}}\left|\hat{v}_{\varepsilon}(t)-\hat{v}_{0}(t)\right|^{2} d t & \leq \int_{|t| \leq t_{\varepsilon}} e^{-2 k|t|} e^{2 k t \varepsilon}\left|\hat{v}_{\varepsilon}(t)-\hat{v}_{0}(t)\right|^{2} d t \\
& \leq 2 \pi^{-1} e^{2 k t_{\varepsilon}} \int_{-\infty}^{\infty} \hat{G}^{2}(t)\left|\hat{v}_{\varepsilon}(t)-\hat{v}_{0}(t)\right|^{2} d t \\
& =2 \pi^{-1} e^{2 k t_{\varepsilon}}\left|\hat{G}\left(\hat{v}_{\varepsilon}-\hat{v}_{0}\right)\right|_{2}^{2} \\
& \leq 2 \pi^{-1} e^{2 k t \varepsilon} K_{1} \varepsilon\left(\left|\hat{v}_{0}\right|_{2}+(\pi / 2)^{1 / 2}\right) \\
& \equiv K_{2} \varepsilon e^{2 k t_{\varepsilon}}
\end{aligned}
$$




$$
\begin{aligned}
\int_{|t|>t_{\varepsilon}}\left|\hat{v}_{\varepsilon}(t)-\hat{v}_{0}(t)\right|^{2} d t & \leq \int_{-\infty}^{\infty}|t|^{2} t_{\varepsilon}^{-2}\left|\hat{v}_{\varepsilon}(t)-\hat{v}_{0}(t)\right|^{2} d t \\
& =t_{\varepsilon}^{-2} \int_{-\infty}^{\infty} \mid t\left(\hat{v}_{\varepsilon}(t)-\left.v_{0}(t)\right|^{2} d t\right. \\
& \leq K_{1} t_{\varepsilon}^{-2} \leq K_{2} t_{\varepsilon}^{-2}
\end{aligned}
$$

where $K_{2}=2 \pi^{-1}\left(\left|\hat{v}_{0}\right|_{2}+(\pi / 2)^{1 / 2}\right)$.

Now consider the equation

$$
y^{2} e^{2 k y}=1 / \varepsilon .
$$

The function $h(y)=y^{2} e^{2 k y}$ is strictly increasing for $y>0$ and $h\left(\mathbf{R}^{+}\right)=\mathbf{R}^{+}$. Then the equation (23) has a unique solution $t_{\varepsilon}$ and $t_{\varepsilon} \rightarrow \infty$ as $\varepsilon \rightarrow 0$. Hence, we have

$$
2(1+k) t_{\varepsilon} \geq 2 \ln t_{\varepsilon}+2 k t_{\varepsilon}=\ln (1 / \varepsilon) .
$$

Letting $\varepsilon<1$, we have

$$
t_{\varepsilon}^{-1} \leq 2(1+k)\left((\ln (1 / \varepsilon))^{-1} .\right.
$$

By (21), (22) and (24), we have

$$
\left|v_{\varepsilon}-v_{0}\right|_{2}^{2} \leq 2 K_{2} t_{\varepsilon}^{-2} \leq K(\ln (1 / \varepsilon))^{-2}
$$

where $K=8(1+k)^{2} K_{2}$ as desired. This completes the proof of the theorem.

Remark. So far, the discussion has been about the boundary value $v(x)=u(x, 0)$. Now, from (3), we have for $v=v_{0}$

$$
\left.u_{0}(x, y)=-(1 / \pi) \int_{-\infty}^{\infty} y v_{0}(\xi) /(x-\xi)^{2}+y^{2}\right) d \xi+A(x, y)
$$

where $A(x, y)$ is the sum of the last two integrals in (3). If we let

$$
u_{\varepsilon}(x, y)=-(1 / \pi) \int_{-\infty}^{\infty} y v_{\varepsilon}(\xi) /\left((x-\xi)^{2}+y^{2}\right) d \xi+A(x, y)
$$


then it can be shown that, as $\varepsilon \rightarrow 0$,

$$
\sup \left|u_{\varepsilon}(\cdot ; y)-u_{0}(\cdot ; y)\right|_{2} \leq K_{3}(\ln (1 / \varepsilon))^{-1}
$$

where $K_{3}$ is a constant depending only on the $H^{1}$-norm of $v_{0}$, and the sup is over $0<y<\phi(x),-\infty<x<\infty$. Details are omitted.

\section{APPENDIX}

Here we calculate the behavior of $(\partial H / \partial n)(x, \phi(x))$ in which $\vec{n}=$ $\left\{-\phi^{\prime}(x) / \alpha,-1 / \alpha\right\}, \alpha=\left[1+\phi^{\prime}(x)^{2}\right]^{1 / 2}$ is the inner unit normal to the curve $y=\phi(x)$ with respect to the domain $D$.

By (3), it suffices to consider the behavior of $(\partial V / \partial n)(x, \phi(x))$ with

$$
\begin{aligned}
V(x, y)= & \int_{-\infty}^{\infty} G_{1}(x, y, \xi, \phi(\xi)) u_{1}(\xi) d \xi \\
= & \frac{1}{2 \pi} \int_{-\infty}^{\infty} \frac{y+\phi(\xi)+(x-\xi) \phi^{\prime}(\xi)}{(x-\xi)^{2}+(y+\phi(\xi))^{2}} u_{1}(\xi) d \xi \\
& +\frac{1}{2 \pi} \int_{-\infty}^{\infty} \frac{y-\phi(\xi)-(x-\xi) \phi^{\prime}(\xi)}{(x-\xi)^{2}+(y-\phi(\xi))^{2}} u_{1}(\xi) d \xi
\end{aligned}
$$

Note that

$$
\begin{aligned}
\frac{d}{d \xi}\left(\arctan \frac{x-\xi}{y+\phi(\xi)}\right) & =-\frac{y+\phi(\xi)+(x-\xi) \phi^{\prime}(\xi)}{(x-\xi)^{2}+(y+\phi(\xi))^{2}} \\
\frac{d}{d \xi}\left(\arctan \frac{x-\xi}{y-\phi(\xi)}\right) & =-\frac{y-\phi(\xi)-(x-\xi) \phi^{\prime}(\xi)}{(x-\xi)^{2}+(y-\phi(\xi))^{2}}
\end{aligned}
$$

Hence if $u_{1}$ is of class $C^{1}$ with $\lim _{|\xi| \rightarrow \infty} u_{1}(\xi)=0$, then integrating by parts, we have

$$
\begin{aligned}
V(x, y)= & \frac{1}{2 \pi} \int_{-\infty}^{\infty} \arctan \frac{x-\xi}{y+\phi(\xi)} u_{1}^{\prime}(\xi) d \xi \\
& +\frac{1}{2 \pi} \int_{-\infty}^{\infty} \arctan \frac{x-\xi}{y-\phi(\xi)} u_{1}^{\prime}(\xi) d \xi
\end{aligned}
$$


Therefore

$$
\begin{aligned}
\alpha \frac{\partial V}{\partial n}(x, y)= & \frac{\partial V}{\partial x}(x, y) \phi^{\prime}(x)-\frac{\partial V}{\partial y}(x, y) \\
= & \frac{1}{2 \pi} \int_{-\infty}^{\infty} \frac{(y+\phi(\xi)) \phi^{\prime}(x)+x-\xi}{(x-\xi)^{2}+(y+\phi(\xi))^{2}} u_{1}^{\prime}(\xi) d \xi \\
& +\frac{1}{2 \pi} \int_{-\infty}^{\infty} \frac{(y-\phi(\xi)) \phi^{\prime}(x)+x-\xi}{(x-\xi)^{2}+(y-\phi(\xi))^{2}} u_{1}^{\prime}(\xi) d \xi .
\end{aligned}
$$

It is easy to see that

$$
\begin{aligned}
\lim \frac{1}{2 \pi} \int_{-\infty}^{\infty} \frac{(y+\phi(\xi)) \phi^{\prime}(x)+x-\xi}{(x-\xi)^{2}+(y+\phi(\xi))^{2}} u_{1}^{\prime}(\xi) d \xi \\
=\frac{1}{2 \pi} \int_{-\infty}^{\infty} \frac{(\phi(x)+\phi(\xi)) \phi^{\prime}(x)+x-\xi}{(x-\xi)^{2}+(\phi(x)+\phi(\xi))^{2}} u_{1}^{\prime}(\xi) d \xi
\end{aligned}
$$

as $(x, y) \rightarrow(x, \phi(x))$ from below.

The second integral in (27) has a singularity when $(x, y) \rightarrow(x, \phi(x))$. We let $h>0$ and note

$$
\begin{aligned}
\lim \frac{1}{2 \pi} \int_{|\xi-x| \geq h} & \frac{(y-\phi(\xi)) \phi^{\prime}(x)+x-\xi}{(x-\xi)^{2}+(y-\phi(\xi))^{2}} u_{1}^{\prime}(\xi) d \xi \\
= & \frac{1}{2 \pi} \int_{|\xi-x| \geq h} \frac{(\phi(x)-\phi(\xi)) \phi^{\prime}(x)+x-\xi}{(x-\xi)^{2}+(\phi(x)-\phi(\xi))^{2}} u_{1}^{\prime}(\xi) d \xi .
\end{aligned}
$$

If we choose $h$ small so $\phi^{\prime}(\xi)-\phi^{\prime}(x)$ and $u_{1}^{\prime}(\xi)-u_{1}^{\prime}(x)$ are sufficiently small, then we have

$$
\begin{aligned}
\frac{1}{2 \pi} \int_{x-h}^{x+h} \frac{(y-\phi(\xi)) \phi^{\prime}(x)+x-\xi}{(x-\xi)^{2}+(y-\phi(\xi))^{2}} u_{1}^{\prime}(\xi) d \xi \\
\simeq \frac{1}{2 \pi} \int_{x-h}^{x+h} \frac{(y-\phi(\xi)) \phi^{\prime}(\xi)+x-\xi}{(x-\xi)^{2}+(y-\phi(\xi))^{2}} u_{1}^{\prime}(x) d \xi \\
=\frac{1}{2 \pi} u_{1}^{\prime}(x)\left[-\frac{1}{2} \ln \left((x-\xi)^{2}+(y-\phi(\xi))^{2}\right)\right]_{x-h}^{x+h} \\
=\frac{1}{4 \pi} u_{1}^{\prime}(x) \ln \frac{h^{2}+(y-\phi(x-h))^{2}}{h^{2}+(y-\phi(x+h))^{2}} \\
\rightarrow \frac{1}{4 \pi} u_{1}^{\prime}(x) \ln \frac{1+(1 / h)^{2}(\phi(x)-\phi(x-h))^{2}}{1+\left(1 / h^{2}\right)(\phi(x)-\phi(x+h))^{2}} \equiv \varepsilon(h) .
\end{aligned}
$$


Since $\lim _{h \rightarrow 0} \varepsilon(h)=0$, we have

$$
\begin{aligned}
\lim \frac{1}{2 \pi} \int_{-\infty}^{\infty} \frac{(y-\phi(\xi)) \phi^{\prime}(x)+x-\xi}{(x-\xi)^{2}+(y-\phi(\xi))^{2}} u_{1}^{\prime}(\xi) d \xi \\
=\frac{1}{2 \pi} \int_{-\infty}^{\infty} \frac{(\phi(x)-\phi(\xi)) \phi^{\prime}(\xi)+x-\xi}{(x-\xi)^{2}+(\phi(x)-\phi(\xi))^{2}} u_{1}^{\prime}(\xi) d \xi
\end{aligned}
$$

as $(x, y) \rightarrow(x, \phi(x))$.

Acknowledgments. We would like to thank the referees for their valuable comments and suggestions. We are, in particular, indebted to one of the referees for pointing out several punctuational and grammatical errors in the first version of the paper.

\section{REFERENCES}

1. L. Amerio, Sui problemi di Cauchy e di Dirichlet per l'equazione di Laplace in due variabili, Atti della Reale Accad. d'Italia 21 (1943), 393-425.

2. J. Douglas, Approximate solution of physically unstable problems, Ecole C.E.A.E.D.F., Paris, 1965.

3. N. Landis, Some problems of the qualitative theory of second order elliptic equations (case of several variables), Russian Math. Surveys 18 (1963), 3-62.

4. R. Lattes and J.L. Lions, Methode de quasi-reversibilite et applications, Dunod, Paris, 1967.

5. M.M. Lavrentiev, On the Cauchy problem for the Laplace equation (in Russian), Isvestia Akad. Nauk 20 (1956), 819-842.

6. C. Pucci, Sui problemi di Cauchy non "ben posti" Rend. Accad. Naz. Lincei 18 (1955), 473-477.

7. W.J. Sternberg and T.L. Smith, The theory of potential and spherical harmonics, The University of Toronto Press, Toronto, Canada, 1952.

8. G. Talenti, Un problema di Cauchy, Ann. Scuola Norm. Sup. Pisa Cl. Sci. 18 (1964), 165-186.

9. — Sui problemi mal posti, Boll. Un. Mat. Ital. 15A (1978), 1-29.

10. A.N. Tikhonov and V.Y. Arsenin, Solutions of ill-posed problems, Winston, Wiley, New York, 1977.

Department of Mathematics, Ho Chi Minh City University, Dai Hoc Tong Hop, 227 Nguyen Van Cu, Quan 5, Ho Chi Minh City, Vietnam 